\title{
Symmetric Chaos in a Local Codimension Two Bifurcation with the Symmetry Group of a Square*
}

\author{
Stella Abreu ${ }^{\dagger}$, Philip Aston ${ }^{\ddagger}$, and Ian Melbourne ${ }^{\ddagger}$
}

\begin{abstract}
We study a codimension two steady-state/steady-state mode interaction with $\mathbb{D}_{4}$ symmetry, where the center manifold is three-dimensional. Primary branches of equilibria undergo secondary Hopf bifurcations to periodic solutions which undergo further bifurcations leading to chaotic dynamics. This is not an exponentially small effect, and the chaos obtained in simulations using DsTool is large-scale, in contrast to the "weak" chaos associated with Shilnikov theory.

Moreover, there is an abundance of symmetric chaotic attractors and symmetry-increasing bifurcations. The local bifurcation studied in this paper is the simplest (in terms of dimension of the center manifold and codimension of the bifurcation) in which such phenomena have been identified. Numerical investigations demonstrate that the symmetric chaos is part of the local codimension two bifurcation. The two-dimensional parameter space is mapped out in detail for a specific choice of Taylor coefficients for the center manifold vector field. We use AUTO to compute the transitions involving periodic solutions, Lyapunov exponents to determine the chaotic region, and symmetry detectives to determine the symmetries of the various attractors.
\end{abstract}

Key words. symmetric chaos, symmetry-increasing bifurcations, local bifurcation theory, mode interaction

AMS subject classifications. 37G40, 37G30, 34C28

DOI. $10.1137 / 040603310$

1. Introduction. In dynamical systems with symmetry, it is possible to obtain chaotic attractors with symmetry on average, where the symmetry of the attractor as a set is greater than the symmetry of the individual points in the attractor. Such symmetric attractors were studied by Chossat and Golubitsky [7], who also identified a mechanism called symmetryincreasing bifurcation, where distinct but symmetrically related chaotic attractors collide to produce an attractor with greater symmetry. (This is related to the notion of crises [17].) Subsequent work on numerical and theoretical aspects of symmetric attractors includes [21, $9,23,2,11,14,20]$.

Hitherto, the notions of symmetric chaos and symmetry-increasing bifurcation have been seen as part of global, rather than local, bifurcation theory. In this paper, we show that a variety of symmetry-increasing bifurcations between chaotic attractors occur in an (at first sight, simple) example in low-codimension, low-dimensional bifurcation theory. In contrast to codimension two bifurcations for nonsymmetric vector fields [18, Chapter 7], the chaotic

\footnotetext{
${ }^{*}$ Received by the editors January 16, 2004; accepted for publication (in revised form) by M. Silber July 13, 2004; published electronically January 14, 2005.

http://www.siam.org/journals/siads/4-1/60331.html

${ }^{\dagger}$ Departamento de Matemática, Universidade Portucalense, R. António Bernardino de Almeida, 541/619, 4200 Porto, Portugal (stella@mail.uportu.pt). The research of this author was supported in part by Fundação para a Ciência e a Tecnologia and Fundo Social Europeu, III Quadro Comunitario de Apoio and by MASIE.

${ }^{\ddagger}$ Department of Mathematics and Statistics, University of Surrey, Guildford GU2 7XH, UK (p.aston@surrey.ac.uk, ism@math.uh.edu).
} 
dynamics is determined at finite order in the Taylor series of the vector field.

The existence of chaotic dynamics determined at finite order in low-codimension bifurcations is not new; such dynamics in codimension one and codimension two bifurcations can be found, for example, in $[1,19,13,24,8]$. However, we note that the center manifold is at least four-dimensional in these references. The center manifold here is three-dimensional and arises in a codimension two bifurcation with real eigenvalues, so in a sense this is the simplest local bifurcation in which chaotic dynamics is determined at finite order.

Perhaps of greater significance is the extent — both in phase space and in parameter spaceto which chaotic dynamics exists in the bifurcation studied in this paper. In phase space, our chaotic attractors are not merely thickenings of quasi-periodic attractors (see Figure 12) and could not possibly be mistaken for such. In parameter space, the chaotic regions are not exponentially thin and can be found even by a casual numerical investigation. This is in contrast to the weak chaos in [18, Chapter 7].

In this paper, we are not interested in the provable aspects of chaotic dynamical systems theory; the large-scale chaos discussed in the previous paragraph is surely well beyond existing methods of proof. Instead, our purpose is to use primarily numerical techniques to demonstrate and investigate the extent of the chaos. Starting with analytic calculations to compute primary branches of equilibria and secondary Hopf bifurcation points, we then use AUTO to path-follow the secondary branches of periodic solutions and compute the tertiary bifurcation points. Using DsTool, it is easily seen that subsequent bifurcations lead to extensive chaotic dynamics, symmetric attractors, and symmetry-increasing bifurcations, as mentioned earlier. To automate the investigation, we use maximal Lyapunov exponents to map out the chaotic region in parameter space and symmetry detectives $[4,10]$ to map out the regions in parameter space in which attractors of various symmetry types exist.

The specific bifurcation that we study is a codimension two steady-state/steady-state mode interaction with $\mathbb{D}_{4}$ symmetry, where two independent steady-state bifurcations coalesce. We assume that there is a simple zero eigenvalue and simultaneously a zero eigenvalue of multiplicity two corresponding to the standard representation of $\mathbb{D}_{4}$. Hence, center manifold reduction leads to a three-dimensional vector field. The action of $\mathbb{D}_{4}$ on the center manifold $\mathbb{R}^{3}$ is defined by

$$
\rho:(x, y, w) \mapsto(-y, x, w), \quad \kappa:(x, y, w) \mapsto(x,-y,-w) .
$$

Thus $(x, y)$ transforms under the standard action of $\mathbb{D}_{4}$, while rotations act trivially on $w$. It follows from standard arguments that there are three primary branches of equilibria with maximal isotropy which we label $\mathbb{Z}_{4}, \mathbb{D}_{1}^{e}$, and $\mathbb{D}_{1}^{v}$. (These are cyclic subgroups generated by $\rho$, $\kappa$, and $\kappa \rho$, respectively. The superscripts $e$ and $v$ stand for "edge" and "vertex," distinguishing the two different types of reflection symmetry of a square - axes through opposite edges and axes through opposite vertices.)

In a mode interaction, the primary branches may undergo secondary bifurcations to branches of mixed-mode solutions. It turns out that all three of the primary branches above may undergo secondary Hopf bifurcations leading to periodic solutions with trivial spatial isotropy and nontrivial spatiotemporal symmetry. In what follows, we disregard the $\mathbb{D}_{1}^{v}$ branch and focus on secondary bifurcations from the $\mathbb{Z}_{4}$ and $\mathbb{D}_{1}^{e}$ branches. There is a scenario where both the $\mathbb{Z}_{4}$ and $\mathbb{D}_{1}^{e}$ branches lose stability to supercritical Hopf bifurcations. The resulting 


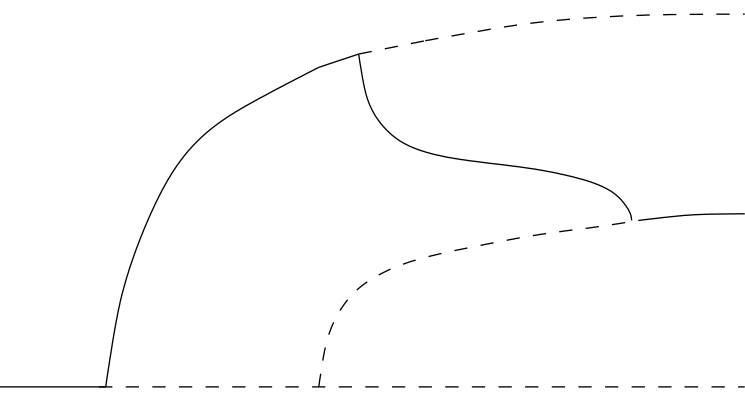

(a)

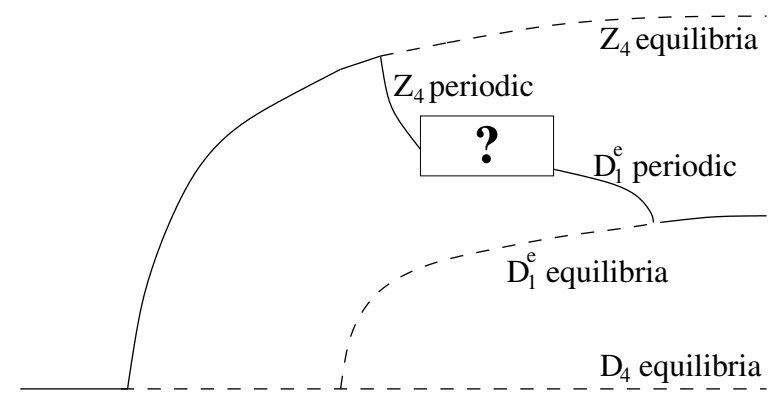

(b)

Figure 1. Schematic bifurcation diagrams for mode interactions in steady-state/steady-state bifurcations with (a) $\mathbb{Z}_{2} \times \mathbb{Z}_{2}$ symmetry, and (b) $\mathbb{D}_{4}$ symmetry. Solid lines denote asymptotically stable solutions, and dashed lines denote unstable solutions.

periodic solutions have $\frac{1}{4}$ and $\frac{1}{2}$ phase shift symmetries, respectively. Since these periodic solutions have different phase shift symmetry, they cannot coalesce, and further tertiary bifurcations are required if the branches are to connect.

The situation up to this point is summarized in Figure 1. In Figure 1(a), we show the "classical" bifurcation diagram for codimension two mode interactions, where there are nonhysteretic transitions between two primary branches of "pure mode" solutions via a secondary branch of mixed-mode solutions. This particular diagram occurs in a steady-state/steady-state bifurcation with $\mathbb{Z}_{2} \times \mathbb{Z}_{2}$ symmetry [15, Figure 4.3(3), Chapter X]. (All branches here consist of equilibria, and, in the usual way, solid lines denote asymptotically stable solutions and dashed lines denote unstable solutions.)

In contrast, Figure 1(b) shows the bifurcation diagram for the steady-state/steady-state bifurcation with $\mathbb{D}_{4}$ symmetry studied in this paper. The mixed-mode branches now consist of periodic solutions, but more significantly their spatiotemporal symmetries are different, and so the bifurcation diagram cannot be complete.

Surprisingly, we find that the missing portion of the bifurcation diagram in Figure 1(b) takes the form of symmetric chaos. Indeed, we compute the existence of attractors with symmetry on average $\mathbb{D}_{1}^{v}$ and $\mathbb{D}_{4}$, as well as asymmetric chaotic attractors, and we compute transitions between these different types of chaotic attractor. (We also find chaotic attractors with symmetry $\mathbb{D}_{1}^{e}$ and $\mathbb{D}_{2}^{e}$, but we do not focus on these here.)

Symmetric chaos has been previously seen in $\mathbb{D}_{4}$ symmetric Takens-Bogdanov bifurcations (codimension two bifurcation, four-dimensional center manifold) [1, 25]. Armbruster, Guckenheimer, and Kim [1] found a "weakly chaotic" symmetric attractor, though the symmetry was not mentioned explicitly. In the same situation, Rucklidge [25] made an extensive study of symmetric chaos and symmetry-increasing bifurcations, though the analysis was restricted to parameters near an $\mathbf{O}(2)$ symmetric limit, so the effective codimension was three.

The mode interaction that we study has been partially analyzed in previous work of Lari-Lavassani et al. [22, section 4.2]. In particular, [22] pointed out that the existence of secondary periodic solutions bifurcating from the $\mathbb{Z}_{4}$ equilibria should be expected on representation-theoretic grounds. There are no general principles that predict the nature of secondary bifurcations from the remaining primary branches, but concrete calculations 
show that both secondary steady-state and Hopf bifurcations can occur depending on the details of the bifurcation problem. These bifurcations and the existence of symmetric chaos and symmetry-increasing bifurcations were not studied in [22].

The remainder of this paper is organized as follows. The analytic computations, which suffice for the primary and secondary bifurcations, are carried out in section 2. Tertiary bifurcations are analyzed using AUTO [12] and DsTool [3] in section 3. In sections 4 and 5, the existence of symmetric chaotic attractors is established by computing Lyapunov exponents and symmetry detectives $[4,10]$. In section 6 , we summarize our results and describe possible future directions.

2. Primary and secondary bifurcations. We begin by writing the general form of the vector field on the center manifold.

Proposition 2.1. The general smooth $\mathbb{D}_{4}$-equivariant mapping $f: \mathbb{R}^{3} \rightarrow \mathbb{R}^{3}$ has the form

$$
\begin{aligned}
& f_{1}(x, y, w)=h_{1}\left(x^{2}, y^{2}, w^{2}\right) x-h_{2}\left(x^{2}, y^{2}, w^{2}\right) w y, \\
& f_{2}(x, y, w)=h_{1}\left(y^{2}, x^{2}, w^{2}\right) y+h_{2}\left(y^{2}, x^{2}, w^{2}\right) w x, \\
& f_{3}(x, y, w)=h_{3}\left(x^{2}+y^{2}, x^{2} y^{2}, w^{2}\right) w+h_{4}\left(x^{2}+y^{2}, x^{2} y^{2}, w^{2}\right) x y\left(x^{2}-y^{2}\right),
\end{aligned}
$$

where $h_{1}, h_{2}, h_{3}, h_{4}$ are smooth real-valued functions.

Proof. This is standard, as in [16].

In this paper, we consider the truncated vector field

$$
\left.\begin{array}{l}
\dot{x}=\left(\lambda-x^{2}+b y^{2}+d w^{2}\right) x-w y, \\
\dot{y}=\left(\lambda-y^{2}+b x^{2}+d w^{2}\right) y+w x, \\
\dot{w}=\left(\mu+c\left(x^{2}+y^{2}\right)-w^{2}\right) w+e x y\left(x^{2}-y^{2}\right) .
\end{array}\right\}
$$

In the usual way, certain (generically nonzero) coefficients can be normalized to \pm 1 , and we have chosen -1 to ensure that certain primary branches are supercritical.

Remark 2.2. For computations of equilibria, it is possible to use singularity theory to find a suitable truncation of these equations. Recent techniques [6] make the application of singularity theory more tractable. For our situation, a computation [5] shows that the bifurcating equilibria are determined by the vector field (2.1) with $d=e=0$. However, this leads to degenerate secondary Hopf bifurcations, necessitating nonzero values for $d$ and $e$.

Isotropy subgroups. The isotropy subgroups are given up to conjugacy by $\mathbb{D}_{4}, \mathbb{Z}_{4}, \mathbb{D}_{1}^{e}, \mathbb{D}_{1}^{v}$, and $\mathbf{1}$, with the following lattice of inclusions:

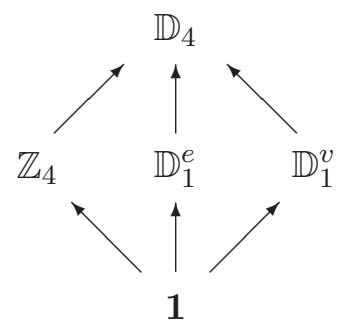

The proper isotropy subgroups $\mathbb{Z}_{4}, \mathbb{D}_{1}^{e}$, and $\mathbb{D}_{1}^{v}$ have one-dimensional fixed-point subspaces:

$$
\operatorname{Fix} \mathbb{Z}_{4}=\{(0,0, w)\}, \quad \operatorname{Fix} \mathbb{D}_{1}^{e}=\{(x, 0,0)\}, \quad \operatorname{Fix} \mathbb{D}_{1}^{v}=\{(x, x, 0)\} .
$$


Primary bifurcations. Table 1 lists the three primary branches of equilibria, showing the branching equations and the eigenvalues for the equilibria.

Table 1

Branching equations and stability assignments for the primary branches of equilibria.

\begin{tabular}{|c|c|c|c|}
\hline $\begin{array}{l}\text { Isotropy } \\
\Sigma\end{array}$ & Fix $\Sigma$ & $\begin{array}{l}\text { Branching } \\
\text { equation }\end{array}$ & Eigenvalues \\
\hline $\mathbb{Z}_{4}$ & $(0,0, w)$ & $\mu=w^{2}$ & $-2 \mu, \quad \lambda+d \mu \pm i \sqrt{\mu}$ \\
\hline $\mathbb{D}_{1}^{e}$ & $(x, 0,0)$ & $\lambda=x^{2}$ & $\begin{array}{ll}-2 \lambda, & \text { eigenvalues of } 2 \times 2 \text { matrix with } \\
& \operatorname{tr}=\mu+(1+b+c) \lambda \\
& \operatorname{det}=\lambda\{(1+b) \mu+((1+b) c-e) \lambda\}\end{array}$ \\
\hline $\mathbb{D}_{1}^{v}$ & $(x, x, 0)$ & $\lambda=(1-b) x^{2}$ & $\begin{array}{ll}-2 \lambda, & \text { eigenvalues of } 2 \times 2 \text { matrix with } \\
& \operatorname{tr}=\mu-2(1+b-c) x^{2} \\
& \operatorname{det}=-2 x^{2}\left\{(1+b) \mu+2((1+b) c-e) x^{2}\right\}\end{array}$ \\
\hline
\end{tabular}

Proposition 2.3. The $\mathbb{Z}_{4}$ equilibria exist for $\mu>0$. They are asymptotically stable if $\lambda+$ $d \mu<0$ and unstable if $\lambda+d \mu>0$.

The $\mathbb{D}_{1}^{e}$ equilibria exist for $\lambda>0$. They are asymptotically stable if

$$
\mu+(1+b+c) \lambda<0, \quad(1+b) \mu+((1+b) c-e) \lambda>0
$$

and are unstable if one or both of these inequalities is reversed.

The $\mathbb{D}_{1}^{v}$ equilibria exist for $\lambda>0$ provided $b<1$, in which case they are asymptotically stable if

$$
(1-b) \mu-2(1+b-c) \lambda<0, \quad\left(b^{2}-1\right) \mu+2(e-(1+b) c) \lambda>0
$$

and are unstable if one or both of these inequalities is reversed.

Secondary bifurcations. Next, we describe the secondary bifurcations of equilibria and periodic solutions that bifurcate from the three primary branches of equilibria.

Table 1 gives information on the eigenvalues associated to the three primary branches of equilibria. Secondary bifurcations occur when the real parts of the eigenvalues vanish. Zero eigenvalues signify steady-state bifurcation to secondary branches of equilibria, and imaginary eigenvalues signify Hopf bifurcation to secondary branches of periodic solutions.

Proposition 2.4. (a) Secondary branches of equilibria bifurcate from the primary branches of equilibria as follows:

$\mathbb{Z}_{4} \quad$ None.

$\mathbb{D}_{1}^{e} \quad$ At $(1+b) \mu+((1+b) c-e) \lambda=0$.

$\mathbb{D}_{1}^{v} \quad$ At $\left(b^{2}-1\right) \mu+2(e-(1+b) c) \lambda=0$.

These secondary bifurcations are pitchfork bifurcations, and the bifurcating equilibria have trivial isotropy.

(b) Secondary branches of periodic solutions bifurcate from the primary branches of equilibria as follows:

$$
\begin{array}{ll}
\mathbb{Z}_{4} & \text { At } \lambda+d \mu=0 . \\
\mathbb{D}_{1}^{e} & \text { At } \mu+(1+b+c) \lambda=0 \text { provided }(1+b)^{2}+e<0 . \\
\mathbb{D}_{1}^{v} & \text { At }(1-b) \mu-2(1+b-c) \lambda=0 \text { provided }-(1+b)^{2}+e>0 .
\end{array}
$$


The resulting periodic solutions have trivial spatial symmetry and spatiotemporal symmetry $\mathbb{Z}_{4}, \mathbb{D}_{1}^{e}$, and $\mathbb{D}_{1}^{v}$, respectively. For example, the $\mathbb{Z}_{4}$ branch has quarter-period phase shift symmetry coupled with the action of $\rho$.

Proof. The eigenvalues for $\mathbb{Z}_{4}$ are given explicitly in Table 1 , so the secondary bifurcations are immediate. The results for $\mathbb{D}_{1}^{e}$ and $\mathbb{D}_{1}^{v}$ are obtained by noting that for a $2 \times 2$ matrix, zero eigenvalues are signified by $\operatorname{det} A=0$ and imaginary eigenvalues are signified by $\operatorname{tr} A=0$, $\operatorname{det} A>0$.

Finally, the results on spatiotemporal symmetry are a consequence of the equivariant Hopf theorem [16].

It follows from general principles that the secondary branches of equilibria and periodic solutions in Proposition 2.4 satisfy "exchange of stability," so their stability is governed by the stability of the primary branch together with the branching direction. Computing the direction of branching is elementary but tedious. We focus on asymptotically stable periodic solutions bifurcating from the $\mathbb{Z}_{4}$ and $\mathbb{D}_{1}^{e}$ branches.

Lemma 2.5. If $b+4 c d<3$, then the $\mathbb{Z}_{4}$ periodic solutions bifurcate supercritically (for $\lambda+d \mu>0)$ and are asymptotically stable. If

$$
(1+b)^{2}+e<0 \text { and }(1+b)\left(2 b^{3}+3 b^{2}-3 b+2 b e+3 b c+2 c e+2 b^{2} c\right)>0,
$$

then the $\mathbb{D}_{1}^{e}$ periodic solutions bifurcate supercritically (for $\mu+(1+b+c) \lambda>0$ ) and are asymptotically stable.

If the appropriate inequality is reversed, then the corresponding periodic solutions exist subcritically and are unstable.

The proof is given in the appendix.

3. Tertiary bifurcations. To compute the loss of stability of the secondary branches of periodic solutions, and the onset of symmetric chaos, it is necessary to use numerical methods. From now on, we specify the values of the constants $b, c, d, e$ in the vector field (2.1) as follows:

$$
b=0.9, \quad c=-2.1, \quad d=-0.05, \quad e=-19.2 .
$$

We concentrate on the positive quadrant $\lambda, \mu>0$ of parameter space. Applying the results of section 2 with these values, we find that the primary branches of equilibria with $\mathbb{Z}_{4}$ and $\mathbb{D}_{1}^{e}$ symmetry are initially asymptotically stable, and each undergo supercritical Hopf bifurcation to secondary branches of periodic solutions with spatiotemporal $\mathbb{Z}_{4}$ and $\mathbb{D}_{1}^{e}$ symmetry. The Hopf bifurcations occur at $\mu=20 \lambda$ and $\mu=0.2 \lambda$, respectively. Primary branches of $\mathbb{D}_{1}^{v}$ equilibria exist but are unstable.

In this section, we use AUTO and DsTool to study the dynamics that occurs when the secondary branches of $\mathbb{Z}_{4}$ and $\mathbb{D}_{1}^{e}$ symmetric periodic solutions lose stability.

Loss of stability of the secondary periodic solutions. In this subsection, we use AUTO [12] to determine curves in $\lambda-\mu$ parameter space where tertiary bifurcations take place from the secondary branches of periodic solutions. At the same time, we determine the manner in which the periodic solutions lose stability.

The use of AUTO is slightly nonstandard due to the spatiotemporal symmetry of the periodic solutions. Standard implementation of AUTO leads to bifurcations that AUTO cannot recognize, and so the approach must be modified as described below. 
$\mathbb{Z}_{4}$ periodic solutions. The $\mathbb{Z}_{4}$ periodic solutions have spatiotemporal symmetry

$$
(x, y, w)(t+T / 4)=\rho \cdot(x, y, w)(t)=(-y, x, w)(t),
$$

where $T$ is the period of the periodic solution.

Solutions with this symmetry type can be computed numerically by constructing a modified Poincaré map $P$. Let $X$ be a local two-dimensional cross-section. Let $g: X \rightarrow \rho X$ be the first hit map and define $P=\rho^{-1} g: X \rightarrow X$. Then periodic solutions with $\mathbb{Z}_{4}$ spatiotemporal symmetry correspond to fixed points of $P$. In practice, we chose $X$ to be contained in the plane $\{w=c\}$ for some constant $c$ and computed $g$ by using an initial value solver to determine the next intersection of the orbit with this plane. Mapping the intersection point back under the action of $\rho^{-1}$ gives the definition of the map $P$ for which AUTO seeks fixed points. Working with $P$ is more efficient numerically than working with the usual Poincaré map since it is sufficient to compute only one quarter of the solution. More significantly, the bifurcations for $P$ are the generic ones, though the consequences for the flow must be interpreted with care; see Remark 3.1 below.

To compute the solutions, we used AUTO to find fixed points of $P$, starting with a solution near the Hopf bifurcation point. By increasing $\lambda$, a path of periodic solutions was computed, and it was found that the solutions on this path lost stability at a turning point. Twoparameter continuation was used to follow the path of turning points in the two-parameter $(\lambda, \mu)$ space shown in Figure 2.

$\mathbb{D}_{1}^{e}$ periodic solutions. The $\mathbb{D}_{1}^{e}$ periodic solutions have spatiotemporal symmetry

$$
(x, y, w)(t+T / 2)=\kappa \cdot(x, y, w)(t)=(x,-y,-w)(t) .
$$

We again consider a local cross-section $X$ and let $g: X \rightarrow \kappa X$ denote the first hit map. Periodic solutions with $\mathbb{D}_{1}^{e}$ symmetry correspond to fixed points for the modified Poincaré map $P=\kappa g: X \rightarrow X$. As in the previous case, we chose $X$ to be contained in the plane $\{w=c\}$ and used a similar approach to construct the map in AUTO.

Using AUTO, we found that the path of stable fixed points for $P$ loses stability via a period-doubling bifurcation. For the underlying flow, this corresponds to a symmetry-breaking pitchfork bifurcation to nonsymmetric periodic solutions. The path of bifurcations is shown in the $(\lambda, \mu)$ plane in Figure 2.

Remark 3.1. We note that this is not a period-doubling bifurcation for the flow and is an example of "suppression of period-doubling" [26]. In fact, the bifurcating nonsymmetric periodic solutions are approximately of the same period as the $\mathbb{D}_{1}^{e}$ periodic solutions near the bifurcation point.

If we had ignored the spatiotemporal symmetry, then the ordinary Poincaré map obtained by integrating around the full periodic solution would have an eigenvalue 1 at the bifurcation point, but the bifurcation is a pitchfork rather than a turning point. This is a highly degenerate bifurcation in systems without symmetry and is not recognized by AUTO.

The pitchfork bifurcation to nonsymmetric periodic solutions turns out to be subcritical, resulting in unstable solutions initially. However, there is almost immediately a turning point at which they regain stability. The corresponding hysteretic region of bistability is extremely thin. The turning point is quickly followed by a period-doubling cascade. We computed the 


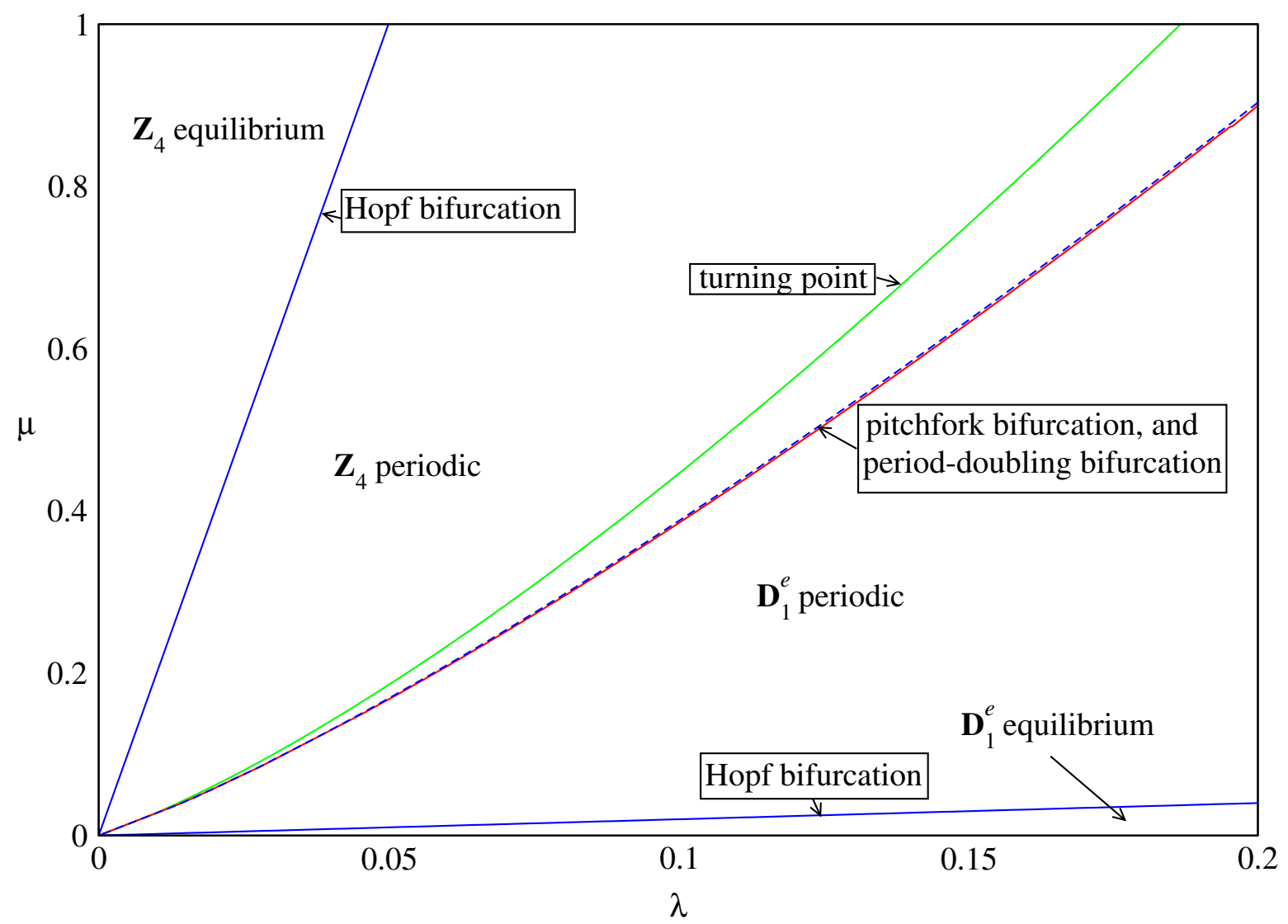

Figure 2. Secondary and tertiary transitions for the vector field $(2.1)$ with $b=0.9, c=-2.1, d=-0.05$, $e=-19.2$.

first period-doubling bifurcation, and the path of bifurcation points is shown as a dashed curve in Figure 2.

To summarize, Figure 2 shows the paths of Hopf bifurcations from primary equilibria to secondary periodic solutions (solid straight lines) and the paths of initial loss of stability of the secondary periodic solutions (solid curves). The thin hysteretic region where stable $\mathbb{D}_{1}^{e}$ periodic solutions and asymmetric periodic solutions coexist is not shown (the curve of turning points where the asymmetric periodic solutions gain stability is omitted), but the dashed curve shows the path of first period-doubling bifurcations for the asymmetric periodic solutions.

Chaotic transitions. In this subsection, we explore the nature of the tertiary transitions using DsTool [3]. Throughout, we fix $\lambda=.16$ and vary $\mu$.

Periodic solutions with spatiotemporal symmetry $\mathbb{D}_{1}^{e}$ and $\mathbb{Z}_{4}$ are shown in Figures 6 and 7. The parameter values are $\mu=.68$ and $\mu=.82$, respectively.

The turning point loss of stability for the $\mathbb{Z}_{4}$ periodic solutions takes place near $\mu=.8186$. Experiments with DsTool indicate that at the turning point there is a hysteretic transition to a $\mathbb{D}_{4}$ symmetric chaotic attractor. Bistability between $\mathbb{Z}_{4}$ symmetric periodic attractors and 
$\mathbb{D}_{4}$ symmetric chaotic attractors occurs for values of $\mu$ roughly in the range $.8186<\mu<.8202$. The $\mathbb{D}_{4}$ symmetric attractor persists until near $\mu=.739$, after which point there is a collapse to a $\mathbb{D}_{1}^{v}$ symmetric chaotic attractor. Varying $\mu$ in the other direction, it can be seen that this is a symmetry-increasing bifurcation from $\mathbb{D}_{1}^{v}$ to $\mathbb{D}_{4}$ symmetric chaos. The $\mathbb{D}_{4}$ and $\mathbb{D}_{1}^{v}$ symmetric attractors for $\mu=.74$ and $\mu=.73$ are shown in Figures 8 and 10. As $\mu$ increases from .73 to .74 , four symmetry-related $\mathbb{D}_{1}^{v}$ symmetric attractors (one of which is shown in Figure 10) collide to form the single fully $\mathbb{D}_{4}$ symmetric attractor shown in Figure 8.

In Figure 9, we show an amalgamation of the $\mathbb{D}_{1}^{e}$ and $\mathbb{Z}_{4}$ periodic solutions from Figures 6 and 7, together with their symmetry-related images. This should be compared with the $\mathbb{D}_{4}$ symmetric chaotic attractor in Figure 8.

Turning to the $\mathbb{D}_{1}^{e}$ periodic solutions, the subcritical pitchfork bifurcation to unstable nonsymmetric periodic solutions occurs at $\mu=.6835$. Asymptotically stable nonsymmetric periodic solutions exist for $.6821 \leq \mu \leq .6869$ with a period-doubling bifurcation at $\mu=.6869$. Experiments with DsTool indicate that a sequence of period-doubling bifurcations follows, leading to a nonsymmetric chaotic attractor at around $\mu=.688$. There is then a symmetryincreasing bifurcation at around $\mu=.6891$ to a fully $\mathbb{D}_{4}$ symmetric chaotic attractor. (We caution the reader that there is a long transient at this parameter value where the attractor appears to have only $\mathbb{D}_{1}^{v}$ symmetry.)

The ensuing region of parameter space seems to be extremely complicated with numerous transitions between periodic/chaotic solutions with/without $\mathbb{D}_{1}^{v}$ symmetry. The transitions take the form of period-doubling sequences, gluing bifurcations and symmetry-increasing bifurcations. Periodic solutions with no symmetry and with $\mathbb{D}_{1}^{v}$ symmetry are shown in Figure 11 .

Eventually, a region of parameter space is reached $(.720 \leq \mu \leq .738)$ where $\mathbb{D}_{1}^{v}$ symmetric chaotic attractors appear to dominate. This region terminates in the aforementioned symmetry-increasing bifurcation to the $\mathbb{D}_{4}$ symmetric attractor.

It is not entirely clear from Figure 8 that the chaotic attractor at $\lambda=0.16, \mu=0.74$ is fully $\mathbb{D}_{4}$ symmetric as claimed. In fact, it is hard to plot a trajectory of sufficient length to resolve this issue. (There are 200,000 data points, but our time step is 0.01, so the total integration time is only 2000 time units.) In Figure 12, we show the same attractor but now in terms of pixels hit by the trajectory. There is no longer a data-storage difficulty, and we use $100,000,000$ data points (corresponding to a total integration time of 1,000,000 time units). The symmetries are now clear. Also, we color the pixels according to how often the pixel is hit. This gives an idea of the density function for the invariant measure.

4. Lyapunov exponents. Our analytic calculations, and the computations using AUTO, leave a large region of parameter space unexplored. The simulations using DsTool indicate that there are chaotic attractors throughout much of this region. To confirm this we computed the maximal Lyapunov exponent for a fixed initial condition (chosen arbitrarily to be $(x, y, w)=$ $(0.008,0.044,0.005))$ and a grid of values of $\lambda$ and $\mu$. Our results are shown in Figure 3.

Most of Figure 3 was obtained by varying $\lambda$ from 0 to 0.2 in increments of 0.002 , and varying $\mu$ from 0 to 1 in increments of 0.01 . To obtain greater resolution near the origin, and to convince ourselves that the chaotic region goes all the way to the origin in parameter space, we zoomed in for $0 \leq \lambda \leq 0.042$, using increments of 0.002 for $\mu$. 


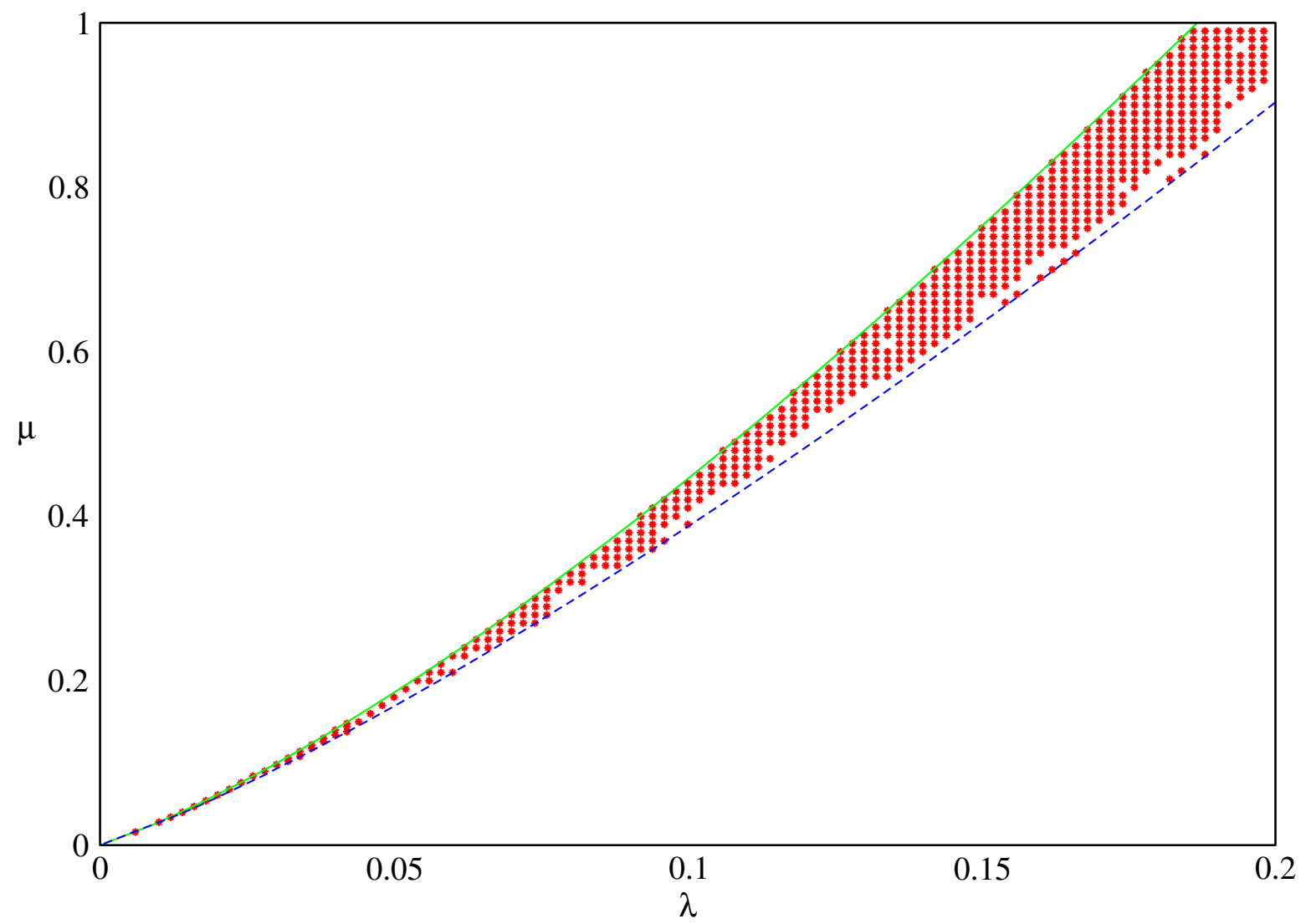

Figure 3. Region of $(\lambda, \mu)$ parameter space with positive Lyapunov exponent for the vector field (2.1) with $b=0.9, c=-2.1, d=-0.05, e=-19.2$. The boundaries of the regions studied analytically and using AUTO (cf. Figure 2) are also shown.

For each point in the $(\lambda, \mu)$ grid, we allowed a transient of 20,000 time units and then computed the maximal Lyapunov exponent over the next 10,000 time units, using a time step of 0.01. The exact numerical values of these finite-time Lyapunov exponents are of course meaningless - for such small values of the parameters, 10,000 time units is unlikely to be sufficient to provide an accurate estimate. Our aim is not to estimate the actual value of the Lyapunov exponents but only to determine whether the dynamics is chaotic or not. For this purpose, we found that 10,000 time units suffice. Indeed, there was a clear cutoff between "positive" and "zero" values of the Lyapunov exponent, with the value 0.001 sufficing. For example, fixing $\lambda=0.1$ and letting $\mu$ vary from 0.38 to 0.45 in increments of 0.01 yield the exponents

$$
\begin{aligned}
& 1.47 \times 10^{-4}, \quad 7.17 \times 10^{-2}, \quad 5.85 \times 10^{-4}, \quad 5.86 \times 10^{-2}, \\
& 7.47 \times 10^{-2}, \quad 9.13 \times 10^{-2}, \quad 1.06 \times 10^{-1}, \quad 2.26 \times 10^{-4},
\end{aligned}
$$

clearly indicating five chaotic parameter values $\mu=0.39,0.41,0.42,0.43,0.44$.

The results we obtain in this way are in perfect agreement with the computations done analytically and with AUTO; see Figure 3. In addition, we have compared the results of the 
Lyapunov exponent calculation with simulations using DsTool (both along slices in parameter space and at certain nonchaotic windows indicated in Figure 3) and found no discrepancies.

5. Symmetry detectives. Symmetry detectives $[4,10]$ are a method for numerically computing the symmetry of an attractor. In general, there are two advantages to proceeding in this way:

(i) For high-dimensional dynamical systems and complicated symmetry groups, it might be hard to determine the symmetries visually.

(ii) The approach can be automated and so can deal with a large number of parameter values, producing a detailed map of parameter space.

In principle, it is not hard to determine the symmetry of a chaotic attractor for the threedimensional vector field (2.1) by looking at the projections into the $(x, y)$ plane. The detective approach is used here primarily because of advantage (ii).

The problem is to determine numerically the symmetries inside $\mathbb{D}_{4}$ that preserve a set $A$ in $\mathbb{R}^{3}$. As shown in Barany, Dellnitz, and Golubitsky [4], this can be done by converting the set $A$ into a point $\psi_{A}$ in some higher-dimensional representation space $V$ for $\mathbb{D}_{4}$ and then computing the symmetry of the point $\psi_{A}$. The key property of $V$ is that every subgroup of $\mathbb{D}_{4}$ should be an isotropy subgroup for the action of $\mathbb{D}_{4}$ on $V$.

We take the representation space $V=\mathbb{R}^{5}$ with coordinates $v=\left(v_{1}, \ldots, v_{5}\right)$, where the action of $\mathbb{D}_{4}$ is defined by

$$
\rho \cdot v=\left(v_{1},-v_{2},-v_{3},-v_{5}, v_{4}\right), \quad \kappa \cdot v=\left(-v_{1}, v_{2},-v_{3}, v_{4},-v_{5}\right) .
$$

Note that $V$ splits up into three distinct nontrivial one-dimensional representations $\mathbb{R}\left\{v_{1}\right\}$, $\mathbb{R}\left\{v_{2}\right\}$, and $\mathbb{R}\left\{v_{3}\right\}$ and the standard two-dimensional representation $\mathbb{R}\left\{v_{4}, v_{5}\right\}$.

Up to conjugacy, there are eight subgroups of $\mathbb{D}_{4}$. Six of these are normal subgroups with fixed-point subspaces in $V$ given by

$$
\begin{aligned}
& \text { Fix } \mathbb{D}_{4}=\{0\}, \quad \text { Fix } \mathbb{D}_{2}^{e}=\mathbb{R}\left\{v_{2}\right\}, \quad \text { Fix } \mathbb{D}_{2}^{v}=\mathbb{R}\left\{v_{3}\right\}, \\
& \text { Fix } \mathbb{Z}_{4}=\mathbb{R}\left\{v_{1}\right\}, \quad \text { Fix } \mathbb{Z}_{2}=\mathbb{R}\left\{v_{1}, v_{2}, v_{3}\right\}, \quad \text { Fix } \mathbf{1}=V .
\end{aligned}
$$

The remaining subgroups $\mathbb{D}_{1}^{e}$ and $\mathbb{D}_{1}^{v}$ each have two conjugate copies (conjugated by $\rho$ ), and the union of fixed-point spaces is a pair of planes in each case:

$$
\text { Fix } \mathbb{D}_{1}^{e}=\mathbb{R}\left\{v_{2}, v_{4}\right\} \cup \mathbb{R}\left\{v_{2}, v_{5}\right\}, \quad \text { Fix } \mathbb{D}_{1}^{v}=\mathbb{R}\left\{v_{3}, v_{4}+v_{5}\right\} \cup \mathbb{R}\left\{v_{3}, v_{4}-v_{5}\right\} \text {. }
$$

These fixed-point spaces are distinct, so each subgroup of $\mathbb{D}_{4}$ is indeed an isotropy subgroup for the action on $V$.

Next, we define the detective

$$
\phi(x, y, w)=(w, x y w, x y, x, y)
$$

It is easy to see that $\phi: \mathbb{R}^{3} \rightarrow V$ is $\mathbb{D}_{4}$-equivariant with respect to the given actions of $\mathbb{D}_{4}$ on $\mathbb{R}^{3}$ and $V$. In addition, $\phi$ is a polynomial map, and each component of $\phi$ is nonzero. Hence $\phi$ satisfies the hypotheses of [4, Theorem 5.2]. 
At this point we switch to the numerically more efficient method developed by Dellnitz, Golubitsky, and Nicol [10]. Given an attractor $A$ with trajectory $u(t)$, we define

$$
\psi_{A}=\lim _{T \rightarrow \infty} \frac{1}{T} \int_{0}^{T} \phi(u(t)) d t
$$

It follows from the ergodic theorem that $\psi_{A} \in V$ is well defined for almost every initial condition $u(0)$, and the point $\psi_{A}$ inherits the symmetry of the set $A$ (under reasonable but technical hypotheses about the ergodic measures on $A$ ). Moreover, it follows from the properties of $\phi$ (being a detective) that typically, the symmetry of $\psi_{A}$ is identical to the symmetry of $A$.

It remains to compute the distance of $\psi_{A}$ from the various fixed-point spaces and hence to determine the symmetry of $A$. Of many possible algorithms, we chose the following:

(i) If $v_{1}^{2}+v_{2}^{2}+v_{3}^{2}+v_{4}^{2}+v_{5}^{2}=0$, then $\mathbb{D}_{4}$.

(ii) Else, if $v_{2}^{2}+v_{3}^{2}+v_{4}^{2}+v_{5}^{2}=0$, then $\mathbb{Z}_{4}$.

(iii) Else, if $v_{1}^{2}+v_{3}^{2}+v_{4}^{2}+v_{5}^{2}=0$, then $\mathbb{D}_{2}^{e}$.

(iv) Else, if $v_{1}^{2}+v_{2}^{2}+v_{4}^{2}+v_{5}^{2}=0$, then $\mathbb{D}_{2}^{v}$.

(v) Else, if $v_{4}^{2}+v_{5}^{2}=0$, then $\mathbb{Z}_{2}$.

(vi) Else, if $v_{1}^{2}>0$, then 1 .

(vii) Else, if $v_{3}^{2}>0$, then $\mathbb{D}_{1}^{v}$.

(viii) Else, $\mathbb{D}_{1}^{e}$.

(In practice, we have to choose the range of values that numerically constitutes zero.)

Our results are shown in Figure 4. These are based on the same initial conditions for $(x, y, w)$ and the same values of $(\lambda, \mu)$ in parameter space that were used in computing Lyapunov exponents in Figure 3. Again, we used time step 0.01 and transient 20,000 time units, but we integrate for the longer time of 100,000 time units. This is necessary to distinguish $\mathbb{D}_{4}$ chaotic attractors from $\mathbb{D}_{1}^{v}$ chaotic attractors near the symmetry-increasing bifurcations. In numerical simulations, the chaos is seen immediately, but it sometimes takes longer to see the fully symmetric attractor.

In Figure 5, we show a blown-up version of part of Figure 4.

6. Conclusions and future directions. In this paper, we have studied a simple-looking codimension two mode interaction with $\mathbb{D}_{4}$ symmetry. The codimension two point is the coalescence of two steady-state bifurcation points, and the center manifold is three-dimensional.

We computed analytically primary branches of equilibria with maximal isotropy giving rise through Hopf bifurcation to secondary branches of periodic solutions with spatiotemporal symmetry.

Surprisingly, we found tertiary bifurcations leading to symmetric chaos. Using AUTO, DsTool, Lyapunov exponents, and symmetry detectives, we obtained convincing numerical evidence that the symmetric chaos is part of the local bifurcation, as is various symmetryincreasing bifurcations between chaotic attractors of differing symmetry types.

There are three natural directions that are worthy of further study:

- The corresponding bifurcations with $\mathbb{D}_{n}$ symmetry for $n \neq 4$ are only partially studied. From now on, we write $\mathbb{D}_{1}$ as shorthand for $\mathbb{D}_{1}^{v}$ and $\mathbb{D}_{1}^{e}$ when $n$ is even and for the unique subgroup of order two (up to conjugacy) when $n$ is odd. The primary branches of equilibria with $\mathbb{Z}_{n}$ and $\mathbb{D}_{1}$ symmetry are essentially unchanged, and there is still a 


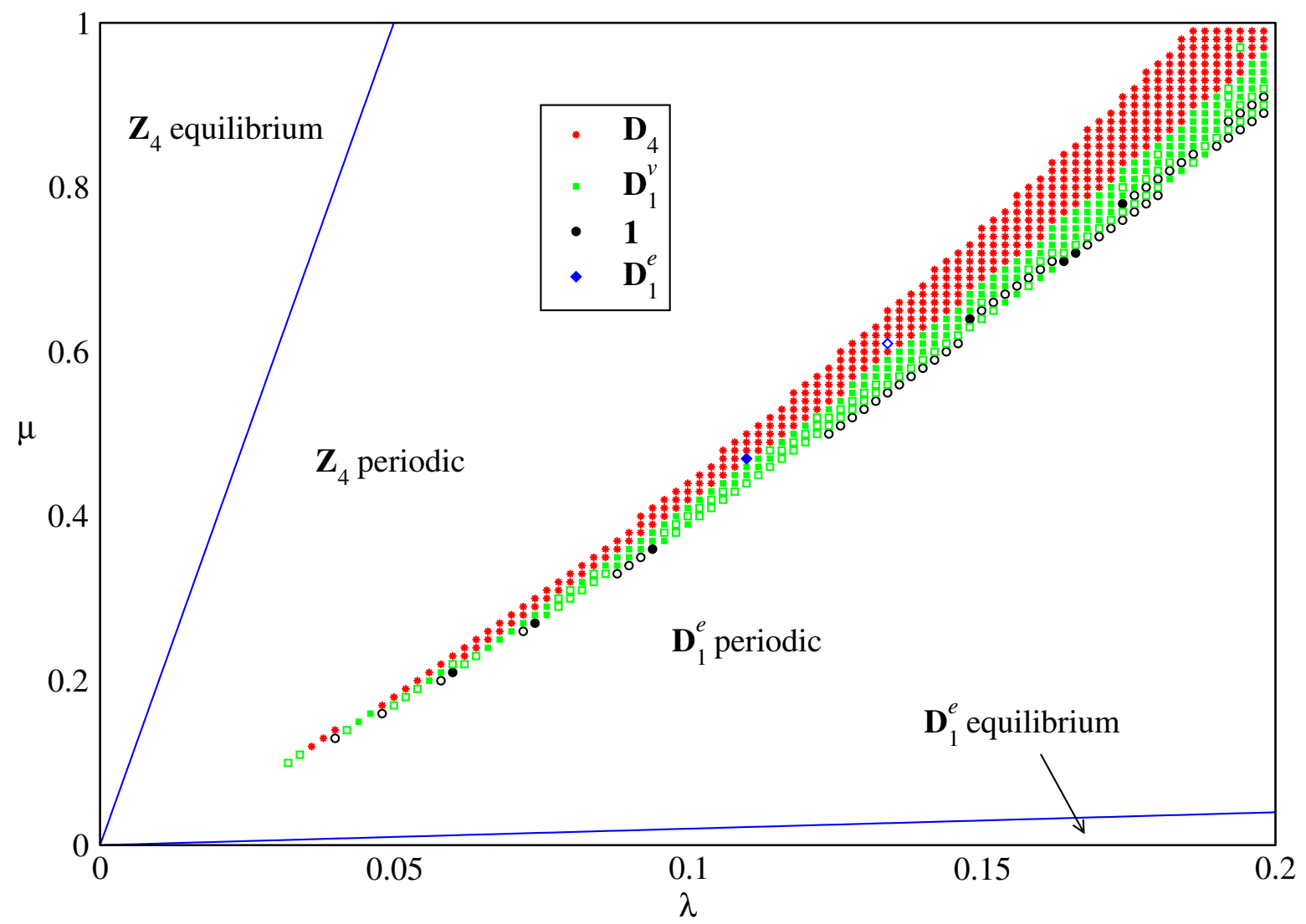

Figure 4. Symmetry types of attractors in $(\lambda, \mu)$ parameter space for the vector field (2.1) with $b=0.9$, $c=-2.1, d=-0.05, e=-19.2$. Solid symbols denote chaotic; clear denote nonchaotic. (The $\mathbb{D}_{4}$ symmetric attractors are all chaotic.)

Hopf bifurcation to a secondary branch of periodic solutions with $\mathbb{Z}_{n}$ spatiotemporal symmetry. However, a calculation for $n \geq 5$ shows that generically the $\mathbb{D}_{1}$ equilibria lose stability only via a steady-state bifurcation, hence producing a secondary branch of asymmetric equilibria. In general, the cases $n \geq 5$ are likely to be simpler than the case $n=4$ studied in this paper, since various important terms are now at higher order. The case $n=3$ is potentially even more complicated than $n=4$ since there are now additional terms at cubic order.

We have not carried out the numerical simulations required to determine the existence of large-scale symmetric chaos and symmetry-increasing bifurcations when $n=3$ and $n \geq 5$.

- It should be straightforward to write a system of reaction-diffusion equations in a square domain undergoing the steady-state/steady-state mode interaction studied in this paper. Hence, it should be possible to realize symmetric chaos and symmetryincreasing bifurcations in a local bifurcation in a system of partial differential equations.

- One implication of our investigations is that symmetric chaos should be more common 


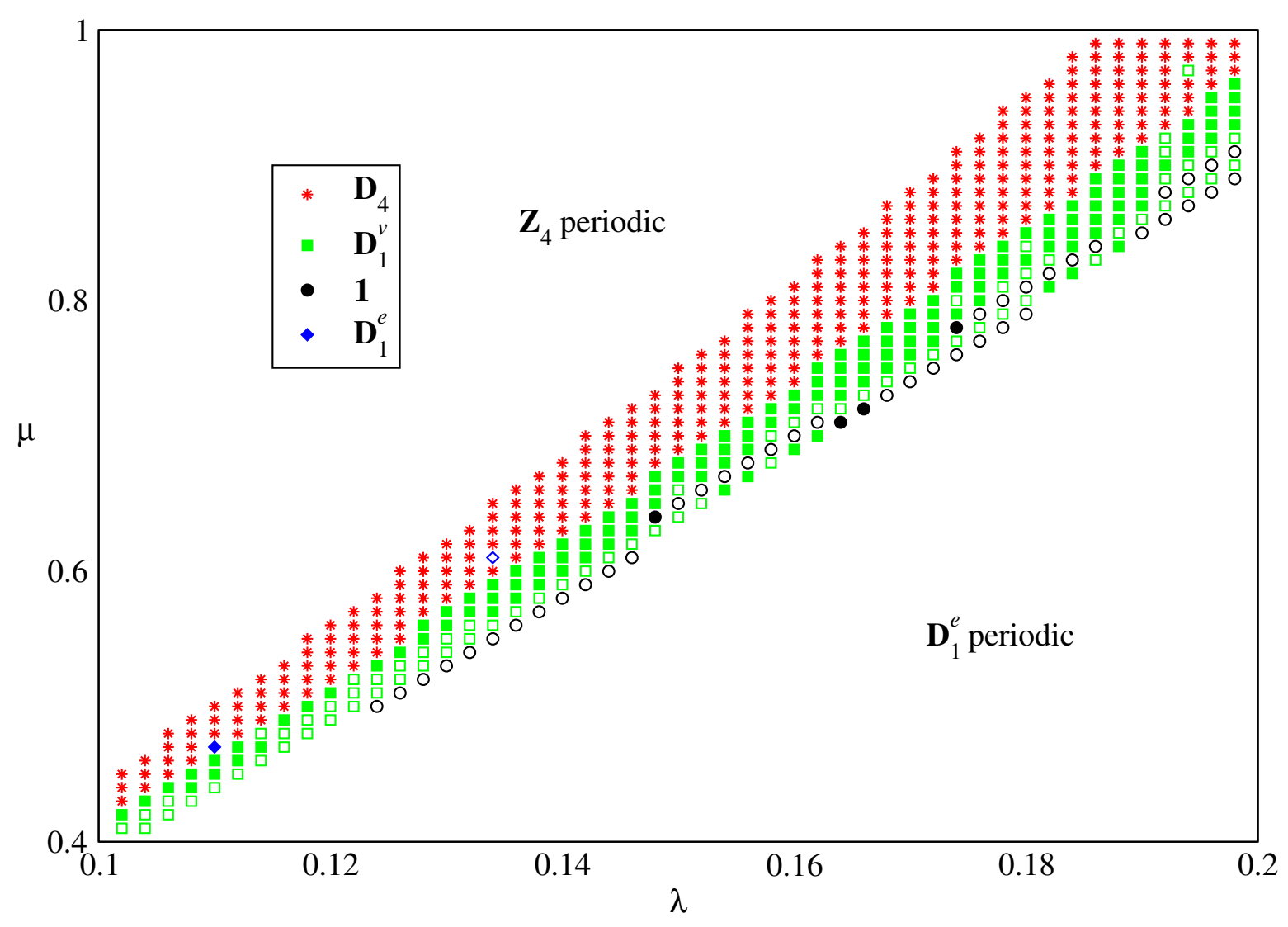

Figure 5. Blow up of subregion of parameter space in Figure 4.

near onset in real experiments than might previously have been anticipated. Likely situations include transitions from a square symmetric equilibrium in an experiment in a square domain but also transitions from a four-fold symmetric equilibrium in an experiment in a circular domain (for example, cellular flames in a circular burner). In the situation of a circular domain, the total dynamics would consist of a rotational drift superimposed on the dynamics described in this paper.

Appendix. Direction of branching for secondary branches of periodic solutions. In this appendix, we give the proof of Lemma 2.5 which establishes the conditions under which the secondary branches of periodic solutions bifurcate supercritically and hence are asymptotically stable.

Direction of branching for $\mathbb{Z}_{4}$ periodic solutions. Setting $\nu=\lambda+d \mu, \widehat{w}=w-\sqrt{\mu}$, and dropping the hats, we obtain

$$
\begin{aligned}
\dot{x} & =\left[\nu-x^{2}+b y^{2}+2 d \sqrt{\mu} w+d w^{2}\right] x-(\sqrt{\mu}+w) y, \\
\dot{y} & =\left[\nu-y^{2}+b x^{2}+2 d \sqrt{\mu} w+d w^{2}\right] y+(\sqrt{\mu}+w) x, \\
\dot{w} & =\left(c\left(x^{2}+y^{2}\right)-2 \sqrt{\mu} w-w^{2}\right)(\sqrt{\mu}+w)+\operatorname{exy}\left(x^{2}-y^{2}\right) .
\end{aligned}
$$


To obtain the center manifold for the secondary bifurcation, write $w=h(x, y)$, where $h$ is at least quadratic. In fact, $h$ is $\mathbb{Z}_{4}$-invariant, so $w=\alpha\left(x^{2}+y^{2}\right)+\cdots$, where we have neglected terms of order 4 or higher. A calculation shows that $\alpha=\frac{c}{2 \sqrt{\mu}}+\cdots$, and the center manifold equations are given by

$$
\begin{aligned}
& \dot{x}=\left[\nu+(c d-1) x^{2}+(b+c d) y^{2}+\cdots\right] x-(\sqrt{\mu}+\cdots) y, \\
& \dot{y}=\left[\nu+(c d-1) y^{2}+(b+c d) x^{2}+\cdots\right] y+(\sqrt{\mu}+\cdots) x .
\end{aligned}
$$

Next we put the equation into Birkhoff normal form. Abstractly, we can write the system as

$$
\begin{aligned}
& \dot{x}=\left(\nu+\alpha x^{2}+\beta y^{2}\right) x-\omega y, \\
& \dot{y}=\left(\nu+\alpha y^{2}+\beta x^{2}\right) y+\omega x .
\end{aligned}
$$

Making the near identity $\mathbb{Z}_{4}$-equivariant change of coordinates $(x, y)=\left(X+\delta Y^{3}, Y-\delta X^{3}\right)$ yields at lowest order

$$
\begin{aligned}
& \dot{X}=\left(\nu+(\alpha+\omega \delta) X^{2}+(\beta-3 \omega \delta) Y^{2}\right) X-\omega Y, \\
& \dot{Y}=\left(\nu+(\alpha+\omega \delta) Y^{2}+(\beta-3 \omega \delta) X^{2}\right) Y-\omega X .
\end{aligned}
$$

Setting $\delta=(\beta-\alpha) /(4 \omega)$ yields

$$
\begin{aligned}
& \dot{X}=\left(\nu+C\left(X^{2}+Y^{2}\right)\right) X-\omega Y, \\
& \dot{Y}=\left(\nu+C\left(X^{2}+Y^{2}\right)\right) Y-\omega X,
\end{aligned}
$$

where $C=\frac{1}{4}(3 \alpha+\beta)=\frac{1}{4}(b+4 c d-3)$. Thus supercritical bifurcation corresponds to $C<0$, yielding the condition $b+4 c d<3$ in Lemma 2.5 .

Direction of branching for $\mathbb{D}_{1}^{e}$ periodic solutions. Setting $\nu=\mu+(1+b+c) \lambda$ and $\widehat{x}=x-\sqrt{\lambda}$ and dropping the hats, we obtain

$$
\begin{aligned}
\dot{x} & =(\sqrt{\lambda}+x)\left(-2 \sqrt{\lambda} x-x^{2}+b y^{2}+d w^{2}\right)-w y, \\
\dot{y} & =y\left((1+b) \lambda-y^{2}+2 b \sqrt{\lambda} x+b x^{2}+d w^{2}\right)+\sqrt{\lambda} w+w x, \\
\dot{w} & =w\left(\nu-(1+b) \lambda+2 c \sqrt{\lambda} x+c x^{2}+c y^{2}-w^{2}\right)+e(x+\sqrt{\lambda}) y\left(x^{2}+2 \sqrt{\lambda} x+\lambda-y^{2}\right) .
\end{aligned}
$$

To obtain the center manifold equations, set $x=\alpha y^{2}+\beta y w+\gamma w^{2}$. Plugging this in and equating coefficients at quadratic order leave the following system of linear equations to be solved for $\alpha, \beta, \gamma$ :

$$
2 \sqrt{\lambda} \alpha+2 \lambda \beta+2 e \lambda^{3 / 2} \gamma=-1, \quad 2(2+b) \sqrt{\lambda} \alpha+e \lambda \beta=b, \quad \sqrt{\lambda} \beta-2 b \lambda \gamma=d \sqrt{\lambda} .
$$

We find

$$
\begin{aligned}
& \alpha=\left\{2 b^{2}+2 b e-d e^{2} \lambda\right\} / 4 \lambda^{1 / 2} \Delta, \\
& \beta=\{-2 b(1+b)+(b+2) d e \lambda\} / 2 \lambda \Delta, \\
& \gamma=\{-2(1+b)+d(e-2 b-4) \lambda\} / 4 \lambda^{3 / 2} \Delta,
\end{aligned}
$$

where $\Delta=b^{2}+2 b+e<0$ (by the hypothesis in Proposition 2.4). 
The equations on the center manifold are given by

$$
\begin{aligned}
& \dot{y}=(1+b) \lambda y+\sqrt{\lambda} w+(2 \sqrt{\lambda} b \alpha-1) y^{3}+(2 \sqrt{\lambda} b \beta+\alpha) y^{2} w \\
&+(2 \sqrt{\lambda} b \gamma+d+\beta) y w^{2}+\gamma w^{3} \\
& \dot{w}=e \lambda^{3 / 2} y+(\nu-(1+b) \lambda) w+e(3 \lambda \alpha-\sqrt{\lambda}) y^{3}+(2 c \sqrt{\lambda} \alpha+c+3 e \lambda \beta) y^{2} w \\
& \quad+(2 c \sqrt{\lambda} \beta+3 e \lambda \gamma) y w^{2}+(2 c \sqrt{\lambda} \gamma-1) w^{3} .
\end{aligned}
$$

Linear algebra plus Birkhoff normal form leads to the normal form equations

$$
\dot{z}=\left(\frac{1}{2} \nu+i \omega\right) z+C|z|^{2} z+\cdots .
$$

Here, $\omega=\lambda \sqrt{-\left((1+b)^{2}+e\right)}$ and $C=\frac{1}{4}(1+b) k \lambda / \Delta+O\left(\lambda^{2}\right)$, where

$$
k=2 b^{3}+3 b^{2}-3 b+2 b e+3 b c+2 c e+2 b^{2} c .
$$

Acknowledgments. We are grateful to Marty Golubitsky for several helpful comments and suggestions and to a referee for instructive criticism. 

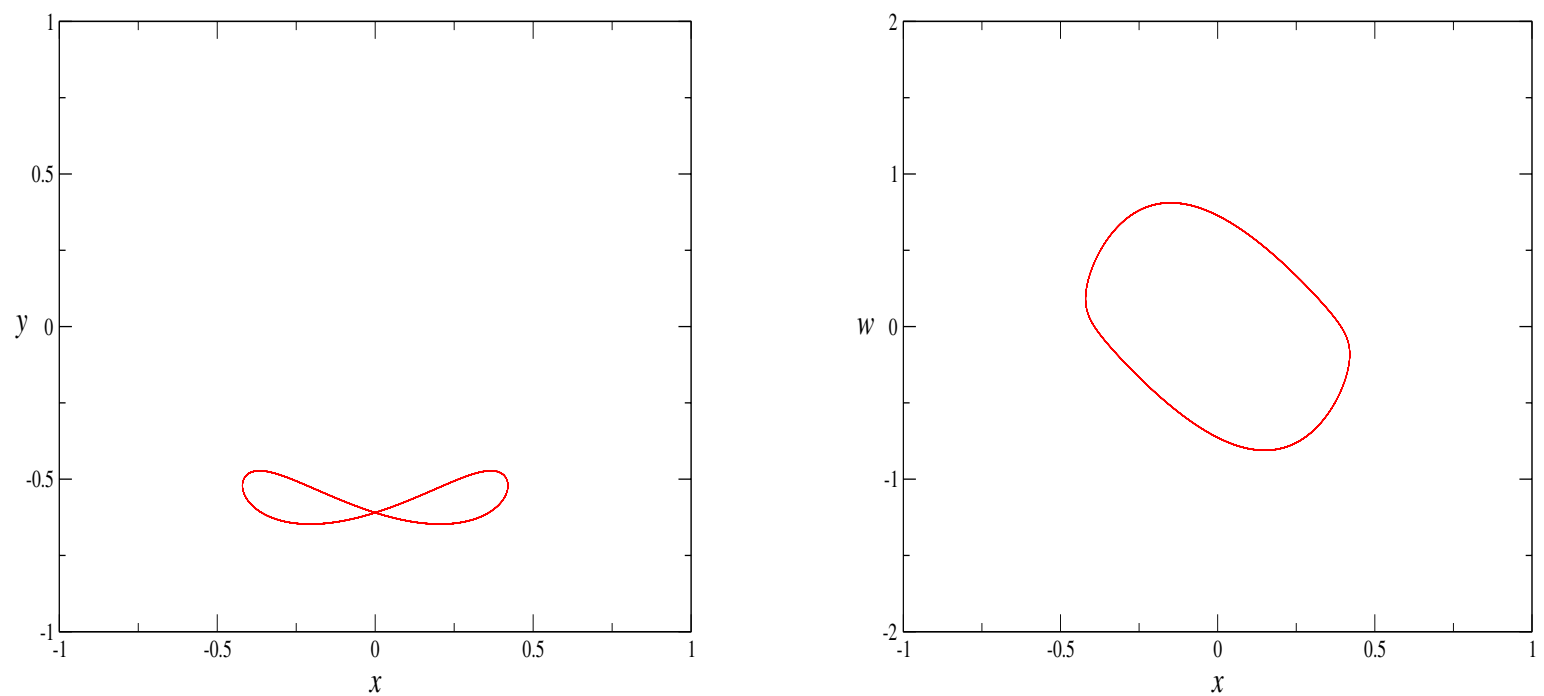

Figure 6. Projection into the $(x, y)$ and $(x, w)$ planes of the $\mathbb{D}_{1}^{e}$ symmetric periodic solution at $\lambda=0.16$, $\mu=0.68$. The plot includes 7,000 data points gathered with time step 0.01 .
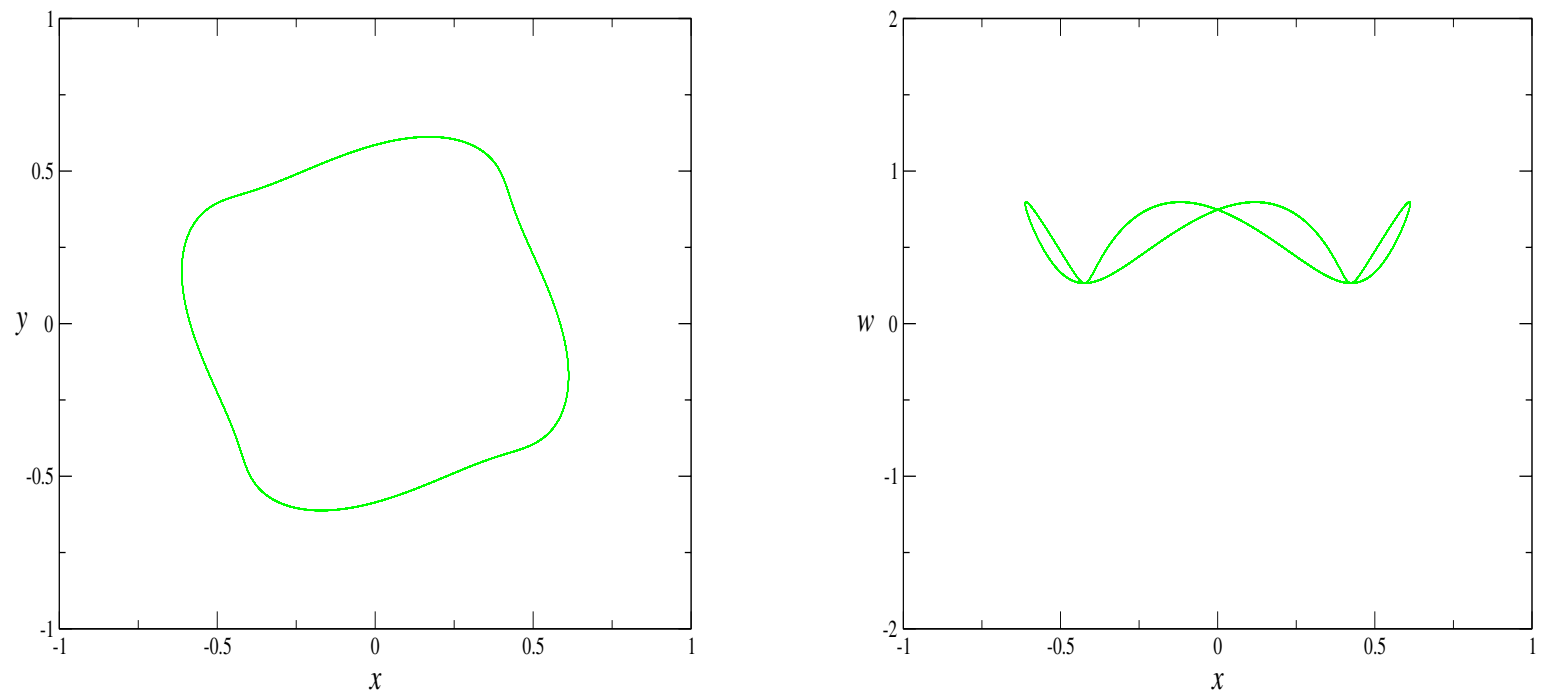

Figure 7. Projection into the $(x, y)$ and $(x, w)$ planes of the $\mathbb{Z}_{4}$ symmetric periodic solution at $\lambda=0.16$, $\mu=0.82$. The plot includes 7,000 data points gathered with time step 0.01 . 

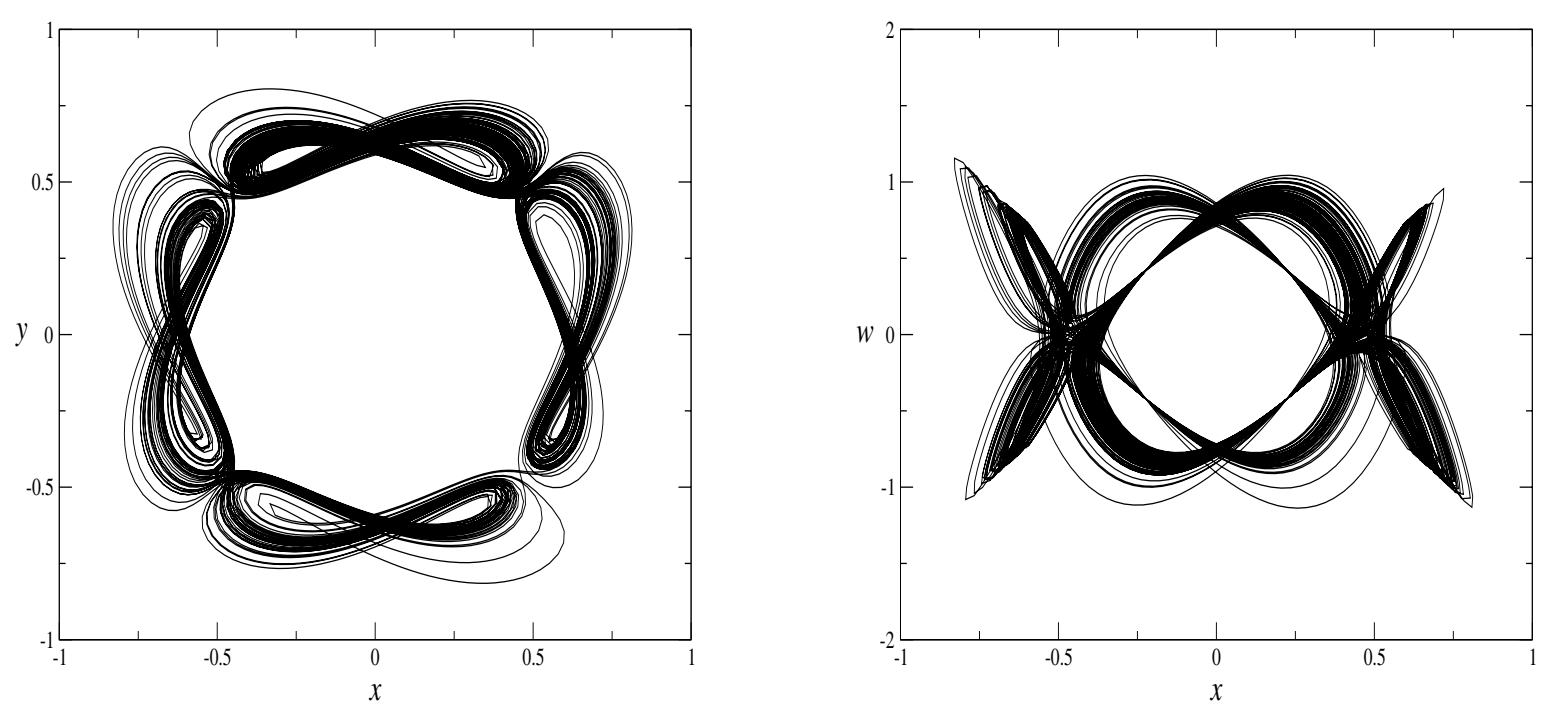

Figure 8. Projection into the $(x, y)$ and $(x, w)$ planes of the $\mathbb{D}_{4}$ symmetric attractor at $\lambda=0.16, \mu=0.74$. The plot includes 200,000 data points gathered with time step 0.01 .
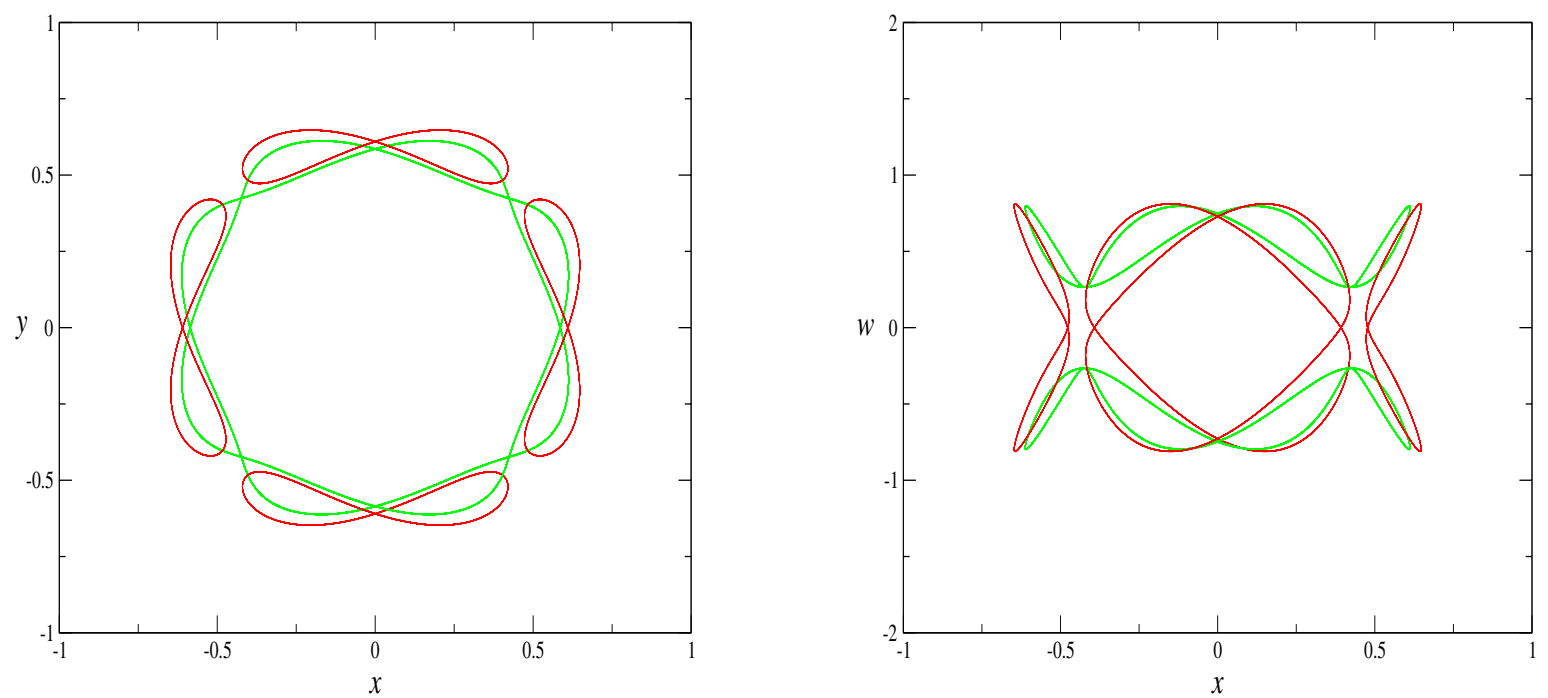

Figure 9. Amalgamation of the plots of the $\mathbb{Z}_{4}$ symmetric and $\mathbb{D}_{1}^{e}$ symmetric periodic solutions shown in Figures 6 and 7, together with their symmetric images. 
50

STELLA ABREU, PHILIP ASTON, AND IAN MELBOURNE
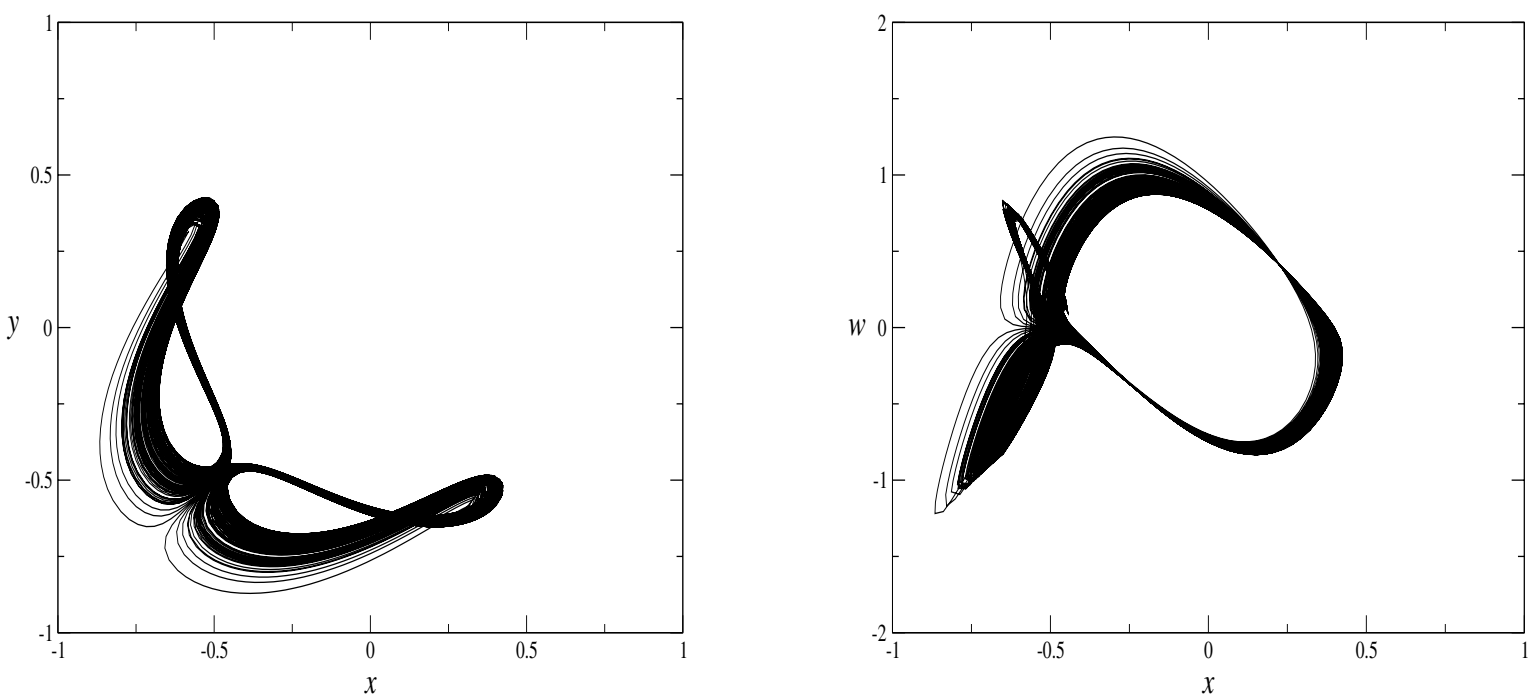

Figure 10. Projection into the $(x, y)$ and $(x, w)$ planes of the $\mathbb{D}_{1}^{v}$ symmetric attractor at $\lambda=0.16, \mu=0.73$. The plot includes 200,000 data points gathered with time step 0.01 .
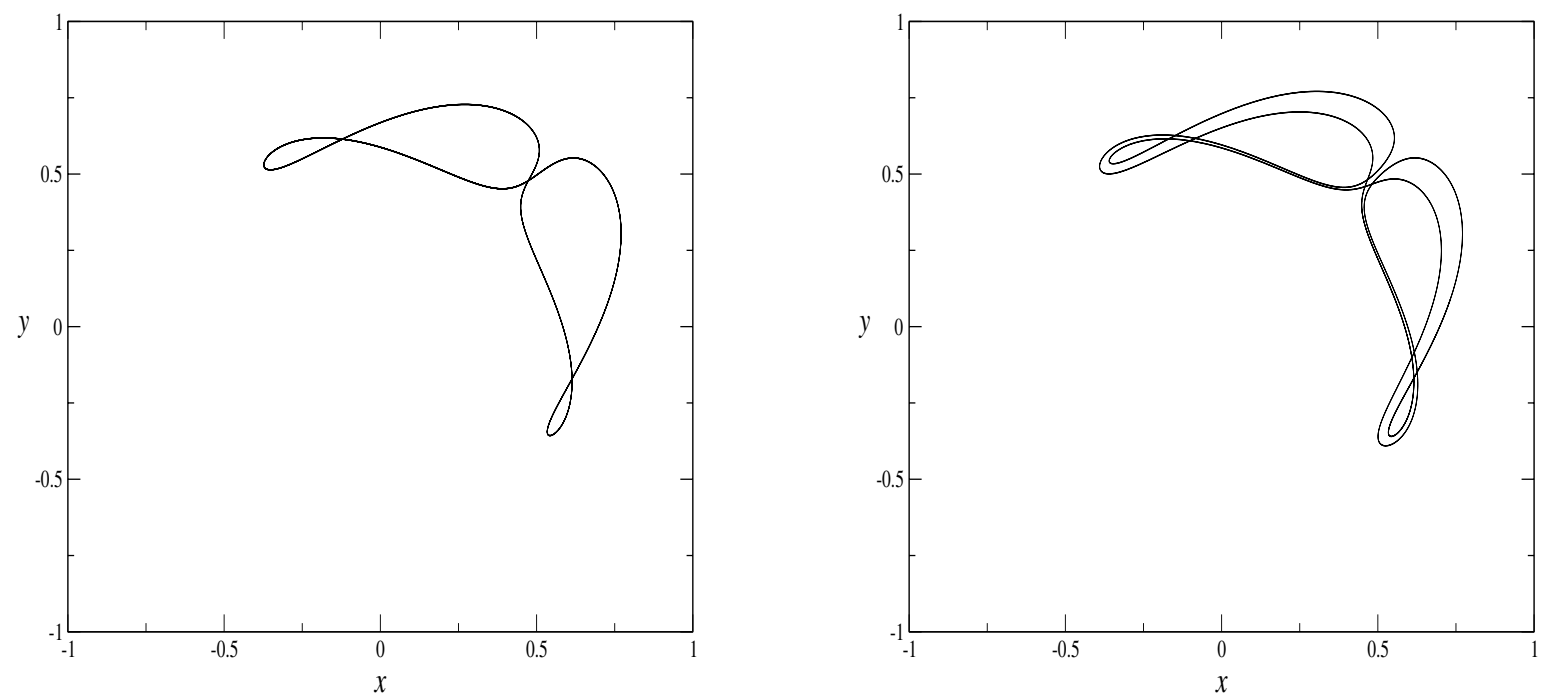

Figure 11. Projection into the $(x, y)$ plane of the asymmetric and $\mathbb{D}_{1}^{v}$ symmetric periodic solutions at $\lambda=0.16, \mu=0.70$ and at $\lambda=0.16, \mu=0.71$. Each plot includes 7,000 data points. 


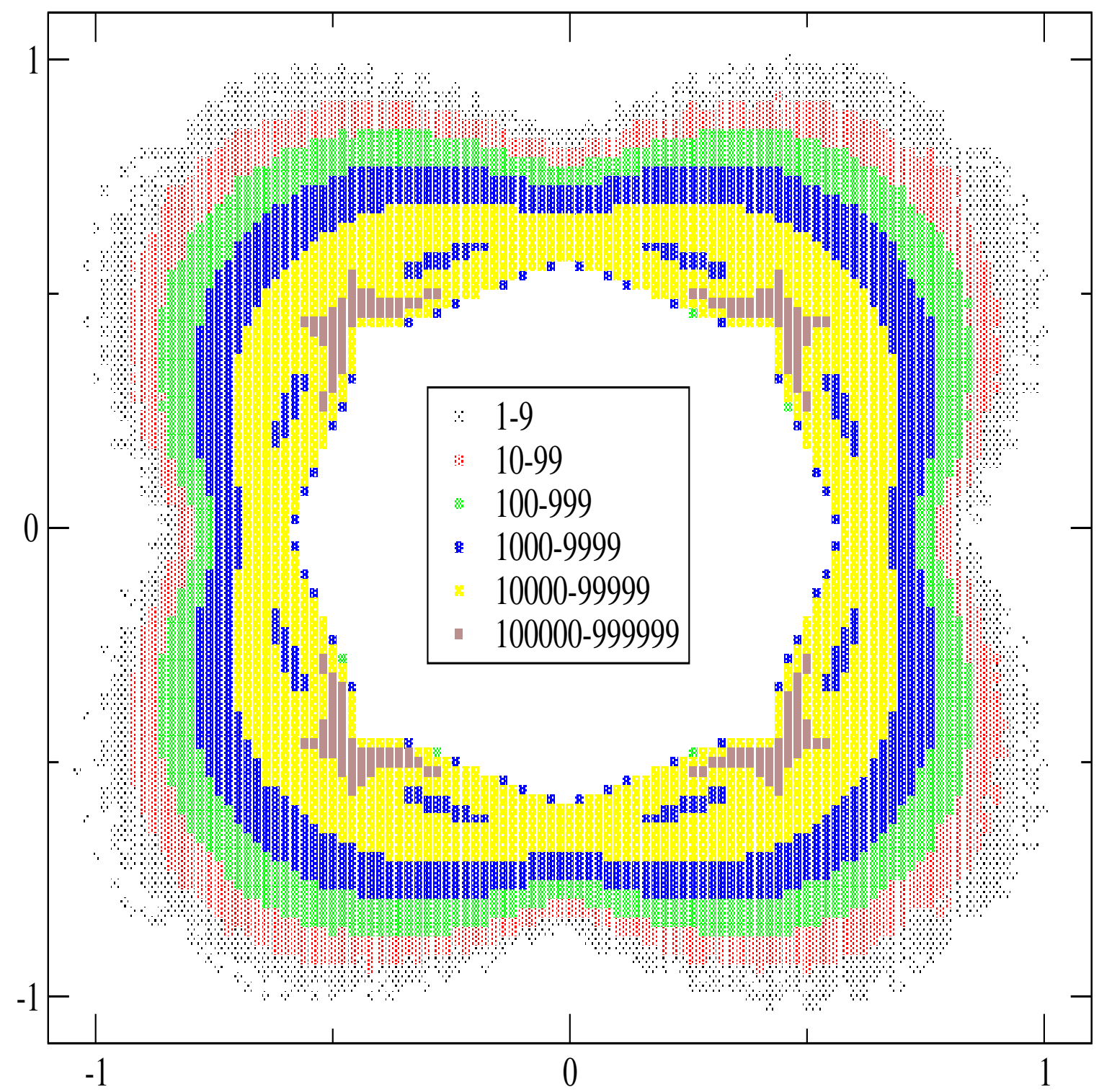

Figure 12. Pixel-based $x y$-plot of the $\mathbb{D}_{4}$ symmetric attractor at $\lambda=0.16, \mu=0.74$, using $100,000,000$ iterates and a grid of $110 \times 110$ pixels of size $.01 \times .01$. A pixel is switched "on" if at least one iterate lands there. The shading is graduated depending on the number of times the pixel is hit. 


\section{REFERENCES}

[1] D. Armbruster, J. Guckenheimer, And S. Kim, Chaotic dynamics in systems with square symmetry, Phys. Lett. A, 140 (1989), pp. 416-420.

[2] P. Ashwin And I. Melbourne, Symmetry groups of attractors, Arch. Ration. Mech. Anal., 126 (1994), pp. 59-78.

[3] A. Back, J. Guckenheimer, M. Myers, F. Wicklin, and P. Worfolk, DsTool: Computer assisted exploration of dynamical systems, Notices Amer. Math. Soc., 39 (1992), pp. 303-309.

[4] E. Barany, M. Dellnitz, and M. Golubitsky, Detecting the symmetry of attractors, Phys. D, 67 (1993), pp. 66-87.

[5] S. B. S. D. CASTro, private communication, 2001.

[6] S. B. S. D. Castro And A. A. DU Plessis, Intrinsic Transversals, manuscript.

[7] P. Chossat And M. Golubitsky, Symmetry-increasing bifurcation of chaotic attractors, Phys. D, 32 (1988), pp. 423-436.

[8] J. H. P. DAWES, A Hopf/steady-state mode interaction in rotating convection: Bursts and heteroclinic cycles in a square periodic domain, Phys. D, 149 (2001), pp. 197-209.

[9] M. Dellnitz, M. Golubitsky, And I. Melbourne, Mechanisms of symmetry creation, in Bifurcation and Symmetry, E. Allgower et al., eds., Internat. Ser. Numer. Math. 104, Birkhäuser, Basel, 1992, pp. 99-109.

[10] M. Dellnitz, M. Golubitsky, And M. Nicol, Symmetry of attractors and the Karhunen-Loève decomposition, in Trends and Perspectives in Applied Mathematics, L. Sirovich, ed., Appl. Math. Sci. 100, Springer-Verlag, New York, 1994, pp. 73-108.

[11] M. Dellnitz And C. HeinRICH, Admissible symmetry increasing bifurcations, Nonlinearity, 8 (1995), pp. 1039-1066.

[12] E. J. Doedel, AUTO, a program for the automatic bifurcation analysis of autonomous systems, Congr. Numer., 30 (1981), pp. 265-384.

[13] M. J. Field, Lectures on Bifurcations, Dynamics and Symmetry, Pitman Res. Notes Math. Ser. 356, Longman, Harlow, UK, 1996.

[14] M. J. Field, I. Melbourne, And M. Nicol, Symmetric attractors for diffeomorphisms and flows, Proc. London Math. Soc., 72 (1996), pp. 657-696.

[15] M. Golubitsky And D. Schaeffer, Singularities and Groups in Bifurcation Theory, Vol. I, Appl. Math. Sci. 51, Springer-Verlag, New York, 1985.

[16] M. Golubitsky, I. N. Stewart, And D. Schaeffer, Singularities and Groups in Bifurcation Theory, Vol. II, Appl. Math. Sci. 69, Springer-Verlag, New York, 1988.

[17] C. Grebogi, E. Ott, F. Romeiras, And J. A. Yorke, Critical exponents for crisis induced intermittency, Phys. Rev. A (3), 36 (1987), pp. 5365-5380.

[18] J. Guckenheimer And P. Holmes, Nonlinear Oscillations, Dynamical Systems, and Bifurcations of Vector Fields, Appl. Math. Sci. 42, Springer-Verlag, New York, 1990.

[19] J. Guckenheimer And P. Worfolk, Instant chaos, Nonlinearity, 5 (1992), pp. 1211-1222.

[20] M. Higuera, J. Porter, And E. Knobloch, Heteroclinic dynamics in the nonlocal parametrically driven nonlinear Schrödinger equation, Phys. D, 162 (2002), pp. 155-187.

[21] G. P. King And I. N. Stewart, Symmetric chaos, in Nonlinear Equations in the Applied Sciences, W. F. Ames and C. F. Rogers, eds., Academic Press, New York, 1991, pp. 257-315.

[22] A. Lari-Lavassani, W. F. Langford, K. Huseyin, and K. Gatermann, Steady-state mode interactions for $D_{3}$ and $D_{4}$-symmetric systems, Dynam. Contin. Discrete Impuls. Systems, 6 (1999), pp. 169-209.

[23] I. Melbourne, M. Dellnitz, And M. Golubitsky, Structure of symmetric attractors, Arch. Ration. Mech. Anal., 123 (1993), pp. 75-98.

[24] J. Porter And E. Knobloch, Complex dynamics in the 1:3 spatial resonance, Phys. D, 143 (2000), pp. $138-168$.

[25] A. M. RuCKLIDGe, Global bifurcations in the Takens-Bogdanov normal form with $D_{4}$ symmetry near the O(2) limit, Phys. Lett. A, 284 (2001), pp. 99-111.

[26] J. W. Swift And K. Wiesenfeld, Suppression of period doubling in symmetric systems, Phys. Rev. Lett., 52 (1984), pp. 705-708. 